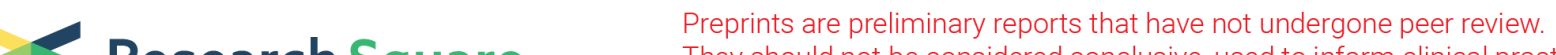 Research Square They should not be considered conclusive, used to inform clinical practice, or referenced by the media as validated information.
}

\section{Modeling Osteoclast Defect and Altered Hematopoietic Stem Cell Niche in Osteopetrosis with Patient-Derived iPSCs}

Inci Cevher Zeytin

Hacettepe University

\section{Berna Alkan}

Hacettepe University: Hacettepe Universitesi

Cansu Ozdemir

Hacettepe University: Hacettepe Universitesi

Duygu Cetinkaya

Hacettepe University: Hacettepe Universitesi

FATMA VISAL OKUR ( $\nabla$ fvokur@hacettepe.edu.tr)

Hacettepe University, Faculty of Medicine https://orcid.org/0000-0002-1679-6205

\section{Research}

Keywords: Osteopetrosis, TCIRG1, iPSC, disease modeling, osteoclast, niche modeling, HSC

Posted Date: March 9th, 2021

DOI: https://doi.org/10.21203/rs.3.rs-258821/v1

License: (c) (1) This work is licensed under a Creative Commons Attribution 4.0 International License. Read Full License 


\section{Abstract \\ Background}

Patients with osteopetrosis present with defective bone resorption caused by the lack of osteoclast activity and hematopoietic alterations, but their bone marrow hematopoietic stem/progenitor cell and osteoclast contents might be different. Osteoclasts recently have been described as the main regulators of HSCs niche, however, their exact role remains controversial due to the use of different models and conditions. Investigation of their role in hematopoietic stem cell niche formation and maintenance in osteopetrosis patients would provide critical information about the mechanisms of altered hematopoiesis. We used patient-derived induced pluripotent stem cells (iPSCs) to model osteoclast defect and hematopoietic niche compartments in vitro.

\section{Methods}

iPSCs were generated from peripheral blood mononuclear cells of patients carrying TCIRG1 mutation. iPSC lines were differentiated first into hematopoietic stem cells-(HSCs), and then into myeloid progenitors and osteoclasts using a step-wise protocol. Then, we established different co-culture conditions with bone marrow-derived hMSCs and iHSCs of osteopetrosis patients as an in vitro hematopoietic niche model to evaluate the interactions between osteopetrotic-HSCs and bone marrowderived MSCs as osteogenic progenitor cells.

\section{Results}

We first demonstrated myeloid-skewed hematopoietic differentiation potential of osteopetrotic iPSCderived hematopoietic progenitors and phenotypically normal and functionally defective osteoclast formation. Upon co-culture with healthy iHSCs, the expression of the genes involved in HSC homing and maintenance (Ang-1, Sdf-1, Jagged-1, N-Cadherine, Kit-L, Opn) in osteopetrotic MSCs which revealed impaired osteogeneic differentiation, as well as their attraction ability over HSCs recovered significantly. Similar change in the phenotype of osteopetrotic iHSCs occured when they interacted with healthy MSCs.

\section{Conclusion}

Our results establish significant alterations in both MSC and HSC compartments of the hematopoietic niche in osteopetrosis patients, which are restored with normal MSC activity supporting the role of defective osteoclasts in all these processes.

\section{Highlights}


- Osteopetrotic osteoclasts phenotypically identical to healthy donor osteoclasts, but functionally defective

- Osteopetrotic-iHSCs have a myeloid-skewed hematopoietic differentiation potential

- Osteopetrotic MSCs shows reduced osteogenic differentiation and their regulatory role in HSC homing and retension is impaired

- Restoration of MSC activity reestablishes normal HSC phenotype and function, supporting the impact of dysfunctional osteoclast in defective niche formation in osteopetrosis

\section{Background}

Osteopetrosis is a rare inherited disease characterized by impaired osteoclast activity causing defective bone resorption and a significant increase in bone mass. Inheritance can be autosomal recessive, dominant, or X-linked, but most severe cases are almost exclusively, autosomal recessive and named as malignant infantile osteopetrosis (MIOP) or autosomal recessive osteopetrosis (ARO). The lack of functional osteoclasts leads to a bone marrow cavity insufficient to support normal hematopoiesis. The resulting extramedullary hematopoiesis gives rise to massive hepatosplenomegaly and macrocephaly. Bony overgrowth results in cranial nerve dysfunction, choanal stenosis, abnormal dentition and developmental delay. Untreated MIOP has a mortality rate of approximately $70 \%$ by six years of age, mostly due to complications related to bone marrow failure. Mutations in several genes have been implicated in the pathogenesis of osteopetrosis, affecting osteoclast development, differentiation (RANK and RANKL; osteoclast-poor form), and function (TCIRG1, CLCN7, OSTM1, CA-II, and PLEKHM1; osteoclast-rich form) [1-3]. Different loss-of-function mutations in T cell immune regulator 1 (TCIRG1) gene which encodes vacuolar (V)-ATPase isoform a3, a subunit of the osteoclast vacuolar proton pump, account for almost $50 \%$ of all children with infantile osteopetrosis, impair osteoclast ruffled-border formation and bone resorption by disturbing secretory lysosome trafficking [4]. Allogeneic hematopoietic stem cell transplantation (HSCT) is the only curative treatment for most children with osteopetrosis, which needs to be done as early as possible before the development of irreversible neurological complications. Transplantation of hematopoietic progenitor cells is intended to reverse the process by providing monocyte-derived donor source of functional osteoclasts, but bone remodeling and establishment of normal hematopoiesis require time. Despite the improvement of transplant strategies and better long-term survival after transplantation, morbidity and mortality rates within the first year after transplantation are still high, with graft failure and early transplant-related complications accounting for most deaths. There is evidence suggesting that the difficulty in achieving engraftment in osteopetrosis is associated with abnormalities in the bone marrow niche and hematopoietic stem cell (HSC) homing $[1,5]$. All patients with osteopetrosis present with defective bone resorption and hematopoietic alterations, but their bone marrow HSC and osteoclast contents might be different. In patients with osteoclast-rich osteopetrosis there is a significant reduction in HSC pool and fibrosis in bone marrow, whereas the presence of some HSC pool has been reported in patients with osteoclast-poor osteopetrosis [6, 7]. There is a definitive need to develop new transplant strategies that will support hematopoiesis to improve engraftment besides providing functional osteoclasts by healthy allografts. Investigation of the 
mechanism of defective osteoclast function and its role in hematopoietic stem cell niche formation and maintenance would be critical to understanding altered hematopoiesis in osteopetrosis.

Hematopoietic stem cells are maintained in the specialized bone marrow niches in which the fate of HSCs with regard to quiescence, proliferation, differentiation and migration is regulated [8, 9]. HSCs reside in the endosteal region, at the interface between the bone and $\mathrm{BM}$, which is the region of active bone remodeling. Enchondral ossification which is essential for the initial formation of hematopoietic stem cell niches in bone marrow is tightly controlled by bone modeling/remodeling involving bone-forming osteoblasts and bone-resorbing osteoclasts $[10,11]$. While the role of osteoblasts located in the endosteal region of bone is well known in the establishment of BM niches, osteoclasts have been mostly studied in HSC mobilization in response to stress or pharmacological stimulants after the establishment of the niche $[9,12-15]$. Recently, osteoclasts have been described as the potential regulators of HSCs niche, besides their bone-resorbing function which provides space for hematopoiesis, since they also provide signals which affect cellular and molecular components of the hematopoietic niche [16]. Osteoclasts regulate the differentiation and function of osteoblasts as well as phenotype of mesenchymal cells, regarded as osteoblast progenitors. Mansour A., et al. demonstrated that osteoclasts are important for the initial niche formation and its colonization by $\mathrm{Lin}^{\text {neg }} \mathrm{Sca}^{+}{ }^{+} \mathrm{CKit}^{+}$(LSK) hematopoietic stem/progenitor cells in osteopetrotic mice (oc/oc mice) through their impact on the mesenchymal compartment. It seems that the role of osteoclasts is much more complex than just providing space for HSC niche. The lack of osteoclast activity in osteopetrosis results in reduced BM hematopoiesis, extramedullary hematopoiesis in the liver and spleen and BM failure. The development of extramedullary hematopoiesis in osteopetrosis also suggests a strong association between HSC niche formation and functional osteoclasts. However, the exact role of osteoclasts remains obscure and controversial due to the use of different models and conditions $[10,17,18]$. For this reason, studying osteoclast biology and its impact on hematopoietic stem cell compartment in bone marrow by using patient-derived osteopetrotic iPSCs have unique opportunities, primarily being an unlimited source of pluripotent stem cells carrying patientspecific disease-causing mutations. This will help to dissect disease pathogenesis and investigate new therapeutic targets through in vitro disease modeling in the laboratory, while paving the way for the development of new transplant strategies to support engraftment in the clinic. The aim of this study is to model the osteoclast defect and to investigate alterations in mesenchymal and HSC compartments of the hematopoietic niche using patient-derived osteopetrotic iPSCs. To our knowledge, modeling osteoclast defect and characterization of HSC compartment and hematopoietic niche using human osteopetrotic iPSCs-derived HSCs and bone marrow-derived mesenchymal stromal cells (BM-MSCs) has not been reported yet.

\section{Materials And Methods}

\section{Generation of Induced Pluripotent Stem Cells from Peripheral Blood Mononuclear Cells}

Frozen peripheral blood mononuclear cells which were isolated by density gradient separation from the peripheral blood samples of one healthy donor and three patients with three different TCIRG1 mutations 
were used in this study (Supplementary Table 1). Immunophenotype of isolated mononuclear cells was evaluated with flow cytometry (CD45 ${ }^{+}$and $\left.C D 34^{\text {neg }}, C D 36^{\text {neg }}, C D 71^{\text {neg }}, C D 235 a^{\text {neg }}\right)$. Erythroid progenitor cells were expanded using Erythroid Expansion Media (EEM) (Stem Cell Technologies), and enrichment of erythroid progenitor cells was demonstrated by flow cytometry on day 10 . The data was analyzed using Beckman Coulter Navios EX Flow Cytometer (Kaluza Analysis 2.1 Software). All antibodies, cytokines, and primer sequences were shown in Table 1.

Erythroid progenitor cells were transduced with Sendai viral vector (SeV) at MOI of 5:5:3 in $1 \mathrm{ml}$ fresh EEM $\left(1 \times 10^{5}\right.$ cell)/well). After spinning at $2250 \mathrm{rpm}$ for 90 minutes at $25^{\circ} \mathrm{C}, 1 \mathrm{ml}$ EEM was added to well and incubated at $37^{\circ} \mathrm{C}$. On day 3, cells were transferred onto Matrigel-coated $10 \mathrm{~cm}$ plate in ReproTSR media (Stem Cell Technologies). Cells were fed with ReproTSR (StemCell Technologies) between day 5 and day 11, then maintained in $\mathrm{TeSR}^{\mathrm{TM}}{ }_{-\mathrm{E}} 8^{\mathrm{TM}}$ (Stem Cell Technologies) medium starting from day 12. iPSC colonies that started to appear around day 12 were harvested with manual microdissection method, transferred into Matrigel-coated dishes, and were expanded with clump passaging method with EDTA [19].

\section{Characterization of iPSC lines}

iPSC lines were characterized by colony morphology, immunofluorescence staining, flow cytometry and qRT-PCR. Detection of residual SeV sequences was evaluated in reprogrammed IPS cells using conventional PCR and verification of the mutations was assessed by sequencing of the genomic locus. Embryoid body formation was performed to show three lineage differentiation potential in vitro. Three iPSC lines were characterized for each sample.

Immunofluorescence staining was performed using PSC 4-Marker Immunocytochemistry Kit (Life Technologies). In brief, after fixation and permeabilization, iPSC colonies were first incubated with primary antibodies (OCT4, SSEA4, SOX2 and TRA1-60) for overnight, and then with conjugated secondary antibodies for $1 \mathrm{~h}$ at RT. Following staining with DAPI, samples were examined under fluorescence microscope (Olympus- IX73).

Immunophenotype of iPSCs was evaluated with flow cytometry (BD Accuri C6 Plus). iPSC colonies were harvested with accutase and single cell suspensions were stained using antibodies against pluripotency markers SSEA4 and OCT4, and erythroid progenitor cell markers CD36 and CD235a.

The mRNA expression levels of the pluripotency-associated genes (Endo-OCT4, Endo-SOX2, NANOG, CMYC, KLF-4, UTF-1, DNMT3b, TERT-1, REX-1, N-Cad,) were analyzed by quantitative real-time PCR (qRTPCR). Total RNA isolation was conducted by Promega, ReliaPrep ${ }^{\text {TM }}$ RNA Cell Miniprep System. qPCR studies were performed using ThermoFisher Maxima SYBER Green qPCR master mix on mic qPCR cycler (Bio Molecular System, v2.6.5). Gene expression was normalized to the expressions of $\beta$-actin. The losses of Sendai virus genome (SeV genome sequence targeted) was assessed by end-point PCR using Promega GoTaq ${ }^{\circledR}$ DNA polymerase. Agarose gel electrophoresis results of end-point PCR analysis were 
presented (Figure S4). The mutation verification after reprogramming was done by sequencing of the target genomic loci in the investigated iPSC lines.

In vitro trilineage differentiation potential of iPSC lines was evaluated with embryoid body (EB) assay. iPSC colonies were harvested using Accutase, single-cell suspensions were prepared at passage 20-24 and cells were seeded onto AggreWell ${ }^{\mathrm{TM}} 800$ plates (StemCell Technologies) in EB formation media (StemCell Technologies) containing $10 \mu \mathrm{M}$ Rock inhibitor (StemCell Technologies). Following a 24-hour suspension culture, EBs were harvested and transferred to ultra-low attachment plates in STEMdiff ${ }^{\text {TM }}$ $\mathrm{APEL}^{\mathrm{TM}} 2$ Medium (StemCell Technologies) and kept in culture for spontaneous differentiation. Differentiated EBs on day 7, day 14, and day 21 were characterized by IF, IHC, and RT-PCR. For immunofluorescence staining, cell aggregates were fixed for 30 minutes with $4 \%$ paraformaldehyde, permeabilized with $1.5 \%$ Triton X-100 for 1 hour, re-fixed in $4 \%$ paraformaldehyde for 15 minutes, then blocked with $2 \%$ goat serum for $3 \mathrm{~h}$, and incubated with primary antibodies overnight at $4^{\circ} \mathrm{C}$ (a-SMA, ab7817; MAP2, ab11267; SOX17, ab192453, Abcam). Aggregates were then washed three times PBS and incubated with secondary antibody (Goat anti-Mouse IgG H\&L (Alexa Fluor 488, abcam, ab150113). Next, aggregates were washed in PBS three times, stained with DAPI for 5 minutes, washed again, and imagined using confocal microscopy (CARLL ZEIS LSM 880).

For immunohistochemistry staining; cell aggregates were dehydrated through a series of graded ethanols, xylene, and paraffin before embedding into paraffin blocks. Five-micron sections, were cut and adhered to charged glass slides. Selected sections were then stained with hematoxylin and eosin. The rest of the sections were deparaffinized, rehydrated, and blocked with endogenous peroxidase, incubated with primary antibodies for one hour, then secondary antibodies for $30 \mathrm{~min}$. After treatment with streptavidin peroxidase, and $D A B$, hematoxylin staining and dehydration, series of graded ethanols and xylene processes were performed, they were imagined using Olympus microscopy (Table S1). Change in the gene expression profile of spontaneously differentiated EBs was assessed on day 7, day 14, and day 21 with RT-PCR using transcripts targetting OCT4 and a-SMA, MAP-2 and SOX17 as lineage-specific markers.

\section{Directed Differentiation of Osteopetrotic Induced Pluripotent Stem Cells to Hematopoietic Precursors and Osteoclasts}

IPS cells at passage 20-24 were differentiated into HSCs using STEMdiff ${ }^{\mathrm{TM}}$ Hematopoietic Kit (StemCell Technologies) using a step-wise differentiation protocol which starts with induction of primitive streak/mesoderm, then hematopoietic specification and followed by hematopoietic cell maturation, myeloid cell expansion and osteoclast lineage differentiation [20]. iPSCs were passaged using EDTA clump passaging method, and 10-20 iPSC aggregates per $\mathrm{cm}^{2}$ were plated on matrigel coated-6 well plate in TeSR ${ }^{T M}$-E8 maintenance medium (StemCell Technologies). The cells were then incubated sequentially in STEMdiff ${ }^{\text {TM }}$ differentiation medium containing different combinations of cytokines and hematopoietic growth factors (hVEGF, hbFGF, IL-6, IL-3, IL-11 and hSCF) for about 10 days. Then a fraction of the cells were harvested with accutase, and the expression of hematopoietic cell markers 
(CD43, CD34, and CD45), and also myeloid cell markers (CD11b, CD14, CD16, CD18, CD38, and CD115) were evaluated to show time-dependent up-regulation of lineage-specific cell surface markers. Rest of the differentiated cells were used in order to continue myeloid-lineage differentiation and to characterize iHSCs following the selection of CD34+ cells with magnetic-activated cell sorting (Miltenyi, Cat \# 130042-201), i.e. methocult assay, immunophenotyping, co-culture experiments, and migration assays. The hematopoietic precursors were cultured for another 4 days in order induce myeloid differentiation using StemSpan SFEM (StemCell Technologies)-based differentiation medium enriched with 2mM Glutamine, 4x10-4 M monothyioglycerol, $50 \mu \mathrm{g} / \mathrm{ml}$ L-ascorbic acid, 10 ng/ml hVEGF, 4 U/ml EPO, 50 ng/ml TPO, 100 $\mathrm{ng} / \mathrm{ml}$ hSCF, $10 \mathrm{ng} / \mathrm{ml}$ hlL-6, $5 \mathrm{ng} / \mathrm{ml}$ hlL-11, and $40 \mathrm{ng} / \mathrm{ml}$ hlL-3 (Supplement Table 1). Then a fraction of cells were harvested and stained with CD11b, CD14, CD16, CD18, CD34, CD38, CD43, CD45, CD115 antibodies to demonstrate the efficacy of myeloid differentiation with flow cytometry. Osteoclast differentiation protocol was applied to the remaining fraction of the cells.

Hematopoietic colony-forming potential of CD34+ iPSC-derived HSCs (iHSC) were assessed with semisolid Methocult cultures (StemCell Technologies, MethoCult ${ }^{\mathrm{TM}} \mathrm{H} 4434$ Classic). $4 \times 10^{4}$ cells were seeded per 35-mm culture dishes in 1,5 $\mathrm{ml}$ of methylcellulose, and cultured for 14 days. Colony types were determined by shape, cell size, and extent of visible erythoid content on Olympous IX73 microscope as BFU-E, CFU-G, CFU-M, CFU-GM, and CFU-GEMM. The colony counts were scored and averaged between duplicates. The myeloid lineage specification and expansion of the cells were confirmed with lineagespecific staining with CD45, CD14, CD16, CD18, CD33, CD36, CD41 by flow cytometry.

At the final step, myeloid precursors were harvested and $1 \times 10^{5}$ cells/well were seeded onto Corning Osteo Assay Surface Microplate (Corning Cat\#3988) in IMDM medium containing $10 \mathrm{ng} / \mathrm{ml}$ M-CSF, $10 \mathrm{ng} / \mathrm{ml}$ RANK-L, and 10\% FBS. They were cultured for 21 days for induction of differentiation into functional osteoclasts. Besides immunophenotyping of the cells with monocyte-macrophage and osteoclast precursor cell markers (CD14, CD16, CD18, CD45, CD51/61), IF staining was performed and cell morphology was evaluated with scanning electron microscope (SEM FEI QUANTA 200F). Gene expression profile of iPSCs derived osteoclasts was also analyzed.

The functionality of iPSC-derived osteoclasts was assessed by the presence of tartrate-resistant acid phosphatase activity (TRAP) activity in the multinucleated cells by immunofluorescence staining. Active functional osteoclasts were also identified by the formation of Filamanteous (F)-actin rings that were demonstrated by immunostaining of actin cytoskeleton with the Phalloidin conjugated to Rhodamine, and secretion of cysteine protease, cathepsin $\mathrm{K}$.

Cathepsin K, NFATC1, Calcitonin R as osteoclast markers genes were analyzed with qRT-PCR at the indicated time points throughout the differentiation. The target gene normalized to the $\beta$-actin endogenous control, $2^{\Delta \Delta \mathrm{CT}}$ value was calculated considering negative control i.e. donor myeloid precursor cells.

\section{Co-culture of hMSCs and iPSC-derived Hematopoietic Stem Cells and Migration Assay}


Mesenchymal stem cells (MSCs) were isolated from bone marrow samples of one healthy donor and three patients with three different TCIRG1 mutations, coculture experiments and migration assays were performed after the characterization of MSCs. The MSCs were characterized by flow cytometry (as $\mathrm{CD} 29^{+}, \mathrm{CD} 44^{+}, \mathrm{CD} 73^{+}, \mathrm{CD} 90^{+}, \mathrm{CD} 105^{+}$and $\left.\mathrm{CD} 34^{\text {neg }}, \mathrm{CD} 45^{\text {neg }}\right)$. Adipogenic and osteogenic differentiation potential of hMSCs were evaluated for each established cell line. For induction of adipocyte differentiation, cells were treated with adipogenic differentiation medium (DMEM LG (Gibco) supplemented with 10\% FBS (Gibco), $1 \mu \mathrm{M}$ dexamethasone (Sigma), $60 \mu \mathrm{M}$ indomethacin (Sigma), $500 \mu$ M 1-methyl-3-isobutylxanthine (IBMX, Sigma), and $5 \mu \mathrm{g} / \mathrm{ml}$ insulin (Sigma-Aldrich) for 21 days. Then they were fixed and stained with Oil Red $\mathrm{O}$ stain to visualize fat droplets within the differentiated cells. For osteoblastic differentiation, cells were treated with osteoblast induction medium (OB) containing DMEMLG (Gibco) supplemented with 10 \% FBS (Gibco), 100 nM dexamethasone (Sigma), $10 \mathrm{mM}$ beta glycerophosphate (Sigma), 0,2 mM ascorbic acid (Sigma) for 21 days, and Alizarin Red staining was performed to signify the osteogenic differentiation.

CD34+ iPSC-derived HSCs $\left(1 \times 10^{4} \mathrm{iHSCs} / \mathrm{cm}^{2}\right)$ enriched with magnetic-active cell sorting were cultured on a confluent layer of bone marrow-derived mesenchymal stem cell (BM-MSCs) in CellGro SCGM supplemented with 10\%FBS, $150 \mathrm{ng} / \mathrm{ml}$ FLT3-L (StemCell Technologies), $150 \mathrm{ng} / \mathrm{ml}$ SCF (StemCell Technologies), and $50 \mathrm{ng} / \mathrm{ml}$ IL-3 (StemCell Technologies) for 4 days. Different co-culture setups were used in order to model the osteopetrotic and healthy hematopoietic niche (donor MSCs-donor iHSCs, patient MSCs-patient iHSCs, donor MSCs-patient iHSCs and patient MSCs-donor-iHSC) by using each patient' or donor's own BM-MSCs and iHSCs, and all conditions were tested as triplicates. After 4 days, co-culture plates were examined to visualize the morphology and location of iHSCs on the MSC layer by phase-contrast microscopy. Both phase-bright cells on the surface of the MSC layer and non-adherent cells were harvested and CD45 negative selection was performed by magnetic-activated cell sorting (Miltenyi) to separate MSCs from iHSCs. Flow cytometry analysis of CD45+ hematopoietic cell fraction was done by using hematopoietic- and myelomonocytic-lineage specific surface markers (CD11b, CD14, CD16, CD18, CD34, CD38, CD43, CD45, CD115) to investigate the effect of osteopetrotic MSCs on the immunophenotype and expansion of iHSCs. CD $45^{\text {neg }}$ cell fraction i.e. MSC was used to investigate the effect of osteopetrotic iHSCs on the expression of genes involved in regulation of HSCs homing and maintenance in the hematopoietic niche; Sdf-1, Jagged-1, N-Cad, Ang-1, Kit-L, Opn with qRT-PCR. Quantification of qRT-PCR signals was performed using the (2- $-\Delta \mathrm{Ct})$ method [21], which calculates relative changes in gene expression of the target gene normalized to the $\beta$-actin endogenous control. The values obtained were represented as the relative quantity of mRNA level variations.

Migration assays were performed to evaluate the regulatory role of MSCs as osteoblast progenitor cells on migration and homing of iHSCs to the hematopoietic niche. $1 \times 10^{6} \mathrm{MSC}$ were plated in the bottom chamber of $5-\mu \mathrm{m}$ pore size transwells (Costar) with MEM-a medium containing $10 \%$ FBS and incubated at $37^{\circ} \mathrm{C}$ and $5 \% \mathrm{CO}_{2}$. After $24 \mathrm{~h}, \mathrm{MSC}$ were labeled with CellTracker ${ }^{\mathrm{TM}}$ Green CMFDA (ThermoFisher), and CD34+ iHSCs labeled with CellTracker ${ }^{\mathrm{TM}}$ Orange CMTMR (ThermoFisher) were plated in transwell inserts. After $4 \mathrm{~h}$ of incubation at $37^{\circ} \mathrm{C}$ and $5 \% \mathrm{CO}_{2}$, remaining cells at the transwell inserts were removed and 
cells migrated to the bottom chambers were visualized and quantified by Olympus IX73 fluorescence microscope.

\section{Statistical analysis}

qRT-PCR, migration, coculture and differentiation assays were performed in three biological replicates for patients and one donor, and qRT-PCR was performed in two replicates. The median values of the patient group and the donor were compared with unpaired two-tailed $T$ test, and patient groups compared each other with paired two-tailed T test. Friedman multiple comparisons test, and One sample Wilcoxon test were used for time-course experiments. Kruskal Wallis multiple comparisons test was applied to independent multiple groups comparisons. Analysis of variance was conducted on the replicate values of experiment groups. P values $<0.05$ were accepted as statistically significant. The data was analyzed using GraphPad Prism 8.01.

\section{Results}

\section{Generation of Induced Pluripotent Stem Cells from Peripheral Blood Erythoid Progenitors of Osteopetrotic Patients}

Peripheral blood mononuclear cells (PBMCs) obtained from three patients with three different mutations of TCIRG1 gene and one healthy donor were enriched for erythroid progenitor cells before reprogramming with CytoTune Sendai reprogramming kit. Enrichment efficiency of erythroid progenitors derived from the patients' samples was confirmed by flow cytometry [CD36 (95,9\% $\pm 1,6), \operatorname{CD} 71(98,5 \% \pm 0,15)$, and CD235a $(72,4 \% \pm 8,78)$ (see Supplementary Figure 1A and 1B)]. The first osteopetrotic iPSC colonies were observed at around day 12-15 after transduction. Eight to ten iPSC colonies from each iPSC line established were picked manually and expanded in culture for detailed analyses (see Supplementary Figure S1-C).

All iPSC lines derived from the osteopetrosis patients and the donor expressed pluripotency markers (SSEA4, OCT3/4, TRA1-60, SOX2) as assessed by immunofluorescence staining (Fig. 1a), and flow cytometry (SSEA4, OCT3/4). More than $90 \%$ of iPSCs were positive for OCT4 (Patients 95,2\% $\pm 1,57$; Donor 92,6\% $\pm 3,55$;), and SSEA4 (Patients 99,07\% $\pm 1,73$; Donor 98,86\% $\pm 0,57$;), while they were either negative or weak positive for CD36 (Patients $4,1 \% \pm 1,73$; Donor $3.06 \% \pm 1.13$ ) and for CD235a (Patients $1,71 \% \pm 0,57$; Donor $1,33 \% \pm 0,55$ ) (Fig. 1b). The pluripotency-associated gene expression profiles were evaluated with a panel of ten different target genes including OCT4, SOX2, c-MYC, KLF4, N-Cad, REX1, TERT1, UTF1, DNMT1, NANOG (see Supplementary Figure 2A). Post-reprogramming mutation verification analysis was also performed and showed that disease-related mutations were retained in all iPSC lines (Fig. 1c).

Tri-lineage differentiation potential of generated iPSC lines were evaluated with embryoid body (EB) formation and ability of iPSC-derived EBs to give rise to early progenitors belonging to each of three germ layers. Spontaneously differentiated EBs were stained triple-positive with lineage-specific MAP2 
(ectoderm, neural lineage marker), a-SMA (mesoderm, mesenchymal stromal cell marker), SOX17 (endoderm, fetal hematopoetic stem cell marker) on day 21 by immunofluorescence staining (Fig. 1d) Lineage-specific gene expression profiles were shown in Supplementary Figure 2B, 2C, 2D.

\section{Osteopetrotic iPSCs Demonstrate Myeloid-Skewed Hematopoietic Differentiation}

Osteopetrotic iPSCs were differentiated into hematopoietic progenitor cells (HPC) using a step-wise protocol. Hematopoietic cell clusters appeared starting from day 3 of culture and progressively increased in number and size up to day 10 finally giving radial-sac like structures. (Fig. 2a). Majority of the multipotent hematopoietic progenitors (> 85\%) co-expressed CD34 and CD45, and myelomonocytic markers albeit at low levels [CD34+CD45+ (Donor 93,6\%; Patients 90,3\% $\pm 2,05$ ), CD11b (Donor 29,4\%; Patients 44,06\% $\pm 3,3$ ), CD14 (Donor 26\%; Patients 22,16\% $\pm 3,7$ ), CD16 (Donor 14\%; Patients 9,9 \% $\pm 1,04$ ), CD18 (Donor 14,9\%; Patients 16,6\% $\pm 1,9$ ), CD38 (Donor $44 \%$; Patients 50,8 \% $\pm 2,6$ ), CD 43 (Donor 90,8\%; Patients $92,9 \% \pm 6,2$ ) (Fig. 2b) Both donor- and osteopetrotic-iHSCs (purity $\geq 94 \%$ after CD34 ${ }^{+}$selection) formed granulocyte/macrophage (CFU-M, CFU-G and CFU-GM), and rare mixed (CFU-GEMM) colonies (Fig. 2C). BFU-E colonies were formed only by donor-iHSCs while osteopetrotic-iHSCs showed a three-fold increase in macrophage colony forming ability (CFU-M). Flow cytometry analysis of hematopoietic colonies revealed the myeloid-skewed hematopoietic differentiation potential of osteopetrotic iHSCs

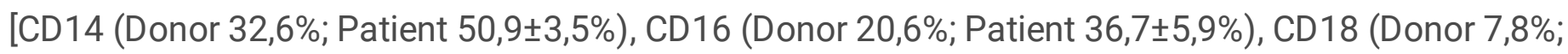
Patient 35,03 $\pm 3,6 \%$ ), CD33 (Donor 81,9\%; Patient 84,2 $\pm 7,4 \%$ ), CD36 (Donor 81\%; Patient $69,13 \pm 3,9 \%$ ), CD41 (Donor 7,9\%; Patient 23,03 $\pm 5,1 \%$ ). Multipotent hematopoietic progenitors were further differentiated into myeloid precurcors by culturing with erytropoietin and thrombopoietin containing differentiation medium for another four days (Fig. 2f). Myeloid-enriched progenitor cells (CD34+CD43+CD45+) was declined from $90,1 \pm 5,1 \%$ to $52,6 \pm 8,9 \%$ following differentiation and expansion and those of expressing monocyte-macrophage lineage-specific markers were increased more significantly by day 14 [ (CD11b from $44 \pm 3,3 \%$ to $60,4 \pm 4,5 \%$ ), CD14 (from $22,2 \pm 3,7 \%$ to $52,9 \pm 0,4 \%$ ), CD16 (from $9,9 \pm 1 \%$ to $55,9 \pm 1 \%$ ), CD18 (from $16,6 \pm 2 \%$ to $46,8 \pm 4,2 \%$ ) ] (Fig. 2e) for patient iHSCs (Paired $T$ test two-tailed $P$ value, $P<0.05$ ). Also, monocyte-macrophage lineage-specific markers expression of patient iHSCs derived myeloid cells were significant higher comparing to healthy donor cells [ CD11b (Donor 42,9; Patient 60,4 $\pm 4,5$ ), CD14 (Donor 38,2; Patient 52,9 \pm 0,4), CD16 (Donor 27,2; Patient 55,9 \pm 1), CD18 (Donor 25,2; Patient 46,8 \pm 4,2)] (Unpaired T test $P$ value, all $P$ values $<0.05$ ).

\section{Osteopetrotic iPSC-derived Myelomonocytic Precursors Generate Dysfunctional Osteoclasts}

We next investigated whether myelomonocytic precursors derived from osteopetrotic iPSCs had the potential to differentiate further into mature osteoclasts. iPSCs-derived myelomonocytic precursors were seeded onto multiple-well plates coated with a synthetic inorganic bone mimetic and cultured in the presence of M-CSF and RANKL, which are essential cytokines for osteoclasts differentiation and survival. Change in cell morphology and size was observed throughout the culture and multinucleated cells were formed at the end of the differentiation period (Fig. 3a). Osteopetrotic-iPSCs-derived multinucleated osteoclasts were phenotypically similar to healthy donor osteoclasts and demonstrated similar 
expressions of CD14 (Donor 98,3\%, Patient 99,1 \pm 0,3\%), CD16 (Donor 98,2\%, Patient 88,3 $\pm 3,7 \%$ ), CD18 (Donor 96,6\%; Patient 99,5 \pm 0,5\%), CD51/61 (Donor 100\%; Patient 78,8 \pm 7,6\%), which are specific surface markers for osteoclast precursors (Fig. 3b). The identity and functionality of differentiated osteoclasts were confirmed by the presence of TRAP activity and secretion of a cysteine protease Cathepsin K, enzymes highly expressed in mature osteoclasts. We further examined the formation of Factin rings which are specific cytoskeletal structures of resorbing active osteoclasts (Fig. 3c). TRAPpositive osteopetrotic-osteoclasts had weak actin ring formation and decreased Cathepsin $\mathrm{K}$ secretion compared to donor osteoclasts. The electron microscope images indicated a difference between the podosome sizes of osteopetrotic and donor multinucleated osteoclasts (Fig. 3c). There was a significant reduction in the expression levels of osteoclast-specific genes; Cathepsin-K, Calcitonin-R, and NFATC1 in osteopetrotic osteoclasts as compared with donor osteoclasts (Fig. 3d). Overall, osteopetrotic osteoclasts which are phenotypically identical to healthy donor osteoclasts were found functionally defective.

\section{Impaired Differentiation Potential, Homing and Retension of Osteopetrotic iHSCs Recover After Co-culture with Healthy MSCs}

Mesenchymal stromal cells are critical components of the hematopoietic niche and they are the progenitors of bone-forming osteoblasts which are required for hematopoietic niche formation. Osteoclasts induce osteoblastic commitment and differentiation of MSCs (Mansour et al. 2012). Thus, alterations in the HSC compartment in the bone marrow of osteopetrosis patients could be related to modifications in MSC compartment. To test this hypothesis, we established a co-culture system with iHSCs and bone marrow-MSCs of osteopetrosis patients to model niche compartments in vitro. First, bone marrow-MSCs were characterized by morphology, immunophenotyping and assessment of their differentiation potency. All MSCs lines expressed specific surface markers of mesenchymal stromal cells and they were negative for hematopoietic markers (see Supplementary Figure 3A). Diminished osteogenic differentiation potential was detected in osteopetrotic BM-MSCs, while they were successfully differentiated into adipocytes (see Supplementary Figure 3B). When immunophenotypes of CD34enriched healthy iHSC co-cultured with healthy MSCs (as in healthy niche) and osteopetrotic iHSCs cocultured with osteopetrotic MSCs (as in osteopetrotic niche) were compared, we found a significant difference among the expressions of myelomonocytic progenitor and differentiation markers, confirming the myeloid-skewed differentiation potential of osteopetrotic iHSCs [CD34+CD43+CD45+ (Donor 27,8\%; Patient 52,1 $\pm 15,4 \%$ ), CD11b (Donor 35,6\%; Patient 66,8 $\pm 9,2 \%$ ), CD14 (Donor 19,7\%; Patient 64,8 $\pm 9,3 \%$ ), CD16 (Donor 8,1\%; Patient 23,6 $\pm 5,45 \%$ ), CD18 (Donor 33,5\%; Patient 81,1 $\pm 8,8 \%$ )] (Unpaired two-tailed T test, all $\mathrm{P}$ values $<0.05$ ) (Fig. 4a). Next, we performed co-culture experiments with iHSCs and MSCs to address the alterations in osteopetrotic iHSCs. We observed an increase in the proportion of $\mathrm{CD} 34^{+}$and $\mathrm{CD} 8^{+}$hematopoietic stem/progenitor cells in osteopetrotic iHSCs co-cultured with healthy MSCs (CD34+ cells; $37,8 \% \pm 9,9$ vs. $52,4 \% \pm 12,15, P>0.05$, CD $38+$ cells; $35,8 \% \pm 5,2$, vs $62,9 \% \pm 1,6 P<0.05)$. The frequency of $\mathrm{CD}_{14}{ }^{+}(64,8 \% \pm 9,3$ vs. $29,03 \% \pm 5,05, \mathrm{P}<0,0001)$ and $\mathrm{CD}_{1} 8^{+}(81,1 \% \pm 8,8$ vs. $63,3 \% \pm 12,4, \mathrm{P}>$ $0,05)$ myeloid progenitors was lower. On the other hand, healthy iHSCs co-cultured with osteopetrotic MSCs showed a myeloid skewed phenotype compared to their phenotype after co-culture with healthy 
MSCs (CD34+CD 43+CD45+, $42,7 \% \pm 2,3$ vs. $27,8 \% \pm 3,1 ; C D 14+, 37,3 \% \pm 2,7$ vs. $19,7 \% \pm 2,9 ; C D 18+, 46,4 \%$ $\pm 3,4$ vs. $33,5 \% \pm 3,2 P<0,05)$.

Altogether these data demonstrate that there is an alteration in the hematopoietic differentiation potential of osteopetrotic HSCs. This alteration could be a rescue mechanism for defective osteoclast function in osteopetrosis. Osteoclasts are tightly coupled with osteoblasts and modulate differentiation and function of osteogenic progenitor cells namely MSCs, and they are essential for healthy niche formation. The restoration of the myeloid-skewed phenotype of osteopetrotic iHSCs after co-culturing with healthy MSCs supports the impairment in osteopetrotic MSCs (Sobacchi, 2013, Villa et al. 2009, Blin-Wakkach et al. 2014).

To gain more insight into the regulatory role of MSCs in HSC niche, we analyzed the expression of genes controlling HSC homing, survival, quiescence and adhesion such as Ang-1, Kit ligand (Kit-L), Sdf-1, Jag-1, Opn and N-Cad in osteopetrotic MSCs after co-culture with osteopetrotic iHSCs. Osteopetrotic MSCs showed lower expression of Ang-1, Jag-1, Sdf-1, Opn, and higher expression of N-Cad when co-cultured with osteopetrotic iHSCs in comparison with the healthy MSCs co-cultured with healthy iHSCs. Interestingly, the expression of Jag-1, Kit-L, and Opn was significantly increased and N-Cad decreased after coculture with healthy iHSCs (Fig. 4b). These results strongly suggest an impairment in MSCs function in supporting HSC homing and maintenance.

Finally, we performed in vitro migration assay to investigate the ability of osteopetrotic MSCs to attract HSCs. A dramatic reduction in attraction ability of osteopetrotic MSCs was observed for both osteopetrotic and healthy iHSCs compared to healthy MSCs. Contrarily, the migratory potential of osteopetrotic- iHCSs was significantly recovered after co-culture with healthy MSCs. (Fig. 4c,and d). Accordingly, these results confirm that altered MSCs do not support HSCs homing and retention in osteopetrosis.

\section{Discussion}

Enchondral ossification which is essential for the initial formation of hematopoietic stem cell niches in bone marrow is tightly controlled by bone modeling/remodeling involving bone-forming osteoblasts and bone-resorbing osteoclasts $[10,11]$. The absence of bone resorption is associated with a decrease in bone marrow space for HSCs as implicated by different osteopetrotic mouse models [11, 22, 23]. But a decrease in bone marrow space is not the only reason of reduced BM hematopoiesis and extramedullary hematopoiesis in osteopetrosis patients since they differ by their osteoclast and HSC contents despite presenting with defective bone resorption [6, 24]. Recently, osteoclasts have been described as the potential regulators of HSCs niche, besides their bone-resorbing function which provides space for hematopoiesis, since they also provide signals which affect cellular and molecular components of the hematopoietic niche [16]. They regulate differentiation and function of mesenchymal cells representing osteoblast progenitors, and MSCs are one of the essential components of HSC niche. Extramedullary hematopoiesis associated with osteopetrosis strongly supports a link between osteoclast function and 
HSC niche [8, 25]. The investigation of the roles of osteoclasts and osteoblasts in HSC niche formation in oc/oc mouse model demonstrated an increased proportion of mesenchymal progenitors with reduced osteoblastic commitment leading to impaired HSC homing which was recovered with the restoration of osteoclast function [10]. Considering clinical heterogeneity in osteopetrosis especially among patients with different mutations, patient-derived iPSCs carrying disease-causing mutation within the context of the patient's whole genome could be an ideal platform to investigate osteoclastogenesis and HSC niche in osteopetrosis [1].

In this study, we reprogrammed peripheral blood mononuclear cells of osteopetrosis patients carrying different mutations in TCIRG1 gene and successfully generated osteoclasts from patient-derived iPSCs. They were phenotypically identical to healthy donor osteoclasts but functionally defective as expected since TCIRG1 mutation affects osteoclast function due to impaired ruffled border formation $[2,26]$. NFATC1 is a master transcription regulator which is responsible from terminal osteoclast differentiation. It promotes osteoclast-specific gene expression which is required for differentiation and bone resorption, such as Calcitonin receptor, Cathepsin K and TRAP [27, 28]. We observed thinner actin ring formation, weak TRAP activity and decreased Cathepsin $\mathrm{K}$ secretion correlated with decreased NFATC1 expression in osteopetrotic-osteoclasts comparing with donor osteoclasts. This data indicates that osteoclasts from patients with TCIRG1 mutation could not differentiate into actively resorbing mature ostoclasts [29]. Interestingly, when patient-derived iPSCs were differentiated into hematopoietic stem/progenitor cells, they showed an increase in macrophage colony-forming ability (CFU-M) compared with healthy donor iHSCs. Further differentiation of osteopetrotic iHSCs into myeloid progenitors gave rise to more monocyte-macrophage progenitors, revealing myeloid-skewed hematopoietic differentiation potential of osteopetrotic iPSCs which leads to enrichment of monocyte-macrophage progenitors. Augmented intraand extramedullary myelopoiesis together with an increase in osteoclastogenesis was reported in oc/oc mice [22]. The upregulation of the myelomonocytic differentiation of osteopetrotic iHSCs may present a compensatory mechanism related to the lack of bone-resorbing active osteoclasts. Since hematopoieis is regulated by bone marrow niche and stress conditions such as irradiation, infections or drugs change heterogeneity of hematopoietic stem/progenitos cells and hematopoietic hierarchy [30,31] myeloid skewing in osteopetrotic iHSCs probably develops as a response to perturbation in the balance between bone-resorbing and bone-forming cells in osteopetrosis.

Osteoclasts control the fate of mesenchymal cells by inducing their differentiation into osteoblasts which is essential for healthy niche formation in bone marrow, as evaluated by the improvement of hematopoiesis after reconstitution of osteoclast activity in osteopetrotic mice $[8,25,32]$. To better understand how defective osteoclast activity can cause alterations in HSC niche contents and impairment of hematopoiesis, we performed hematopoietic niche modeling with an ex vivo co-culture system of iPSC-derived HSCs and bone marrow-MSCs of osteopetrosis patients carrying TCIRG1 mutation.

We demonstrated that osteopetrotic MSCs had reduced osteogenic differentiation potential, and expressed Ang-1, Sdf-1, Jag-1, Opn, the genes associated with HSC homing and maintenance in bone 
marrow at low levels [32-36]. Furthermore, their ability to attract HSCs dramatically impaired. The decreased expression of main regulators of HSC proliferation, quiescence, and homing in osteopetrotic MSCs as well as their impaired attraction ability over HSCs are most likely the mechanisms behind defective HSC homing, and loss of hematopoietic progenitors in bone marrow of osteopetrosis patients. Interestingly, $\mathrm{N}$-cadherin expressed in bone marrow stromal progenitors that are essential for reservation of HSCs in niche especially under stress, was found overexpressed in osteopetrotic MSCs. This could be a stress response to impaired hematopoiesis in osteopetrosis in order to support HSC maintenance in bone marrow niche [37]. In addition, the expression of Jag-1, Kit-L, and Opn in osteopetrotic MSCs was significantly increased and N-Cad decreased upon co-culture with healthy iHSCs. These results strongly suggest a functional impairment in MSCs regarding their role in HSC homing and maintenance and confirm their role in defective hematopoietic niche formation in osteopetrosis through their interaction with dysfunctional osteoclasts. The restoration of function of osteopetrotic MSCs and altered phenotype of osteopetrotic hematopoietic progenitors after co-cultured with their healthy counterparts point out that osteoclasts play a key role in all these modifications. This data may be a proof of concept for clinical studies which evaluate the feasibility/safety of MSC co-transplantation in osteopetrosis patients undergoing allogeneic hematopoietic stem cell transplantation in order to facilitate engraftment through their support on the recovery of abnormal bone marrow niche and HSC homing.

\section{Conclusion}

Our results establish the existence of significant alterations in both MSC and HSC compartments of hematopoietic niche in osteopetrosis patients. Restoration of osteopetrotic MSCs function and altered phenotype of osteopetrotic hematopoietic progenitors upon co-culture with their healthy counterparts point out the regulatory role of osteoclasts in hematopoietic niche. Modeling of osteoclastogenesis and hematopoietic niche compartments with patient-derived iPSCs improve our knowledge about abnormal hematopoiesis in osteopetrosis and provide a research platform to investigate new therapeutic targets while paving the way for the development of new transplant strategies in clinic.

\section{Abbreviations}

iPSC: induced pluripotent stem cells; iHSC: iPSC-derived Hematopoietic stem cells; HSC: Hematopoietic stem cell; MSC: Mesenchymal stem cell, Ang-1: Angiopoietin; Sdf-1: Stromal derived factor 1; Opn: osteopontin; N-cad: N- cadherin; TCIRG1: T Cell Immune Regulator 1; TRAP: Tartrate-resistant acid phosphatase; DMEM-LG:Dulbecco's modified Eagle's medium- Low glucose; NFATC1: Nuclear factoractivated T cells; SeV: Sendai viral vector; CFU: colony forming unit. AP: Alkaline Phosphatase; ARO:Autosomal Recessive Osteopetrosis; BM-MSC: Bone Marrow-Mesenchymal Stem Cell; c-MYC:c-Myc binding protein (MYCBP) pseudogene; cDNA: Complementary DNA; DNMT3B: DNA methyltransferase 3 beta; EB: Embryoid body; EDTA: Ethylenediaminetetraacetic acid; Endo-Oct4 : Endogenous OCT4; EndoSox2: Endogenous SOX2; FBS: Fetal Bovine Serum; H\&E:Hematoxylin-eosin; HSCT: Hematopoietic Stem Cell Transplantation; IBMX : 1-methyl-3-isobutylxanthine; KLF4: Kruppel like factor 4; MIOP : Malignant 
Infantile Osteopetrosis; MOI: Multiplicity of infection; mRNA: Messenger RNA; NANOG: Nanog Homeobox; OCT3/4: Octamer-binding transcription factor 4; PCR: Polymerase Chain Reaction; REX1: RNA exonuclease 1 homolog pseudogene; RNA: Ribonucleic acid; RT-PCR: Real-Time PCR; qPCR: Quantitative PCR; qRT-PCR: Quantitative real-time PCR; SEM: Standard error of the mean; SOX2: SRY-box 2; SSEA4:Stage-specific embryonic antigen-4; TERT: Telomerase reverse transcriptase; UTF1: Undifferentiated embryonic cell transcription factor 1

\section{Declarations}

\section{Acknowledgements}

We are thankful to Jean J. Kim, Ph.D. (Director) for providing expertise and Human Stem Cell Core of Baylor College of Medicine for continuous technical support. We also thank Dr. Aytekin Akyol for his assistance with H\&E and immunohistochemical stainings.

\section{Author contributions}

F.V.O., D.U.C. conceived the project; F.V.O., I.C. designed experiments and analyzed the data. I.C., B.A., C.O. performed the experiments. F.V.O. and D.U.C. designed the patient sample collection and collected the patient samples. All authors approved the final draft and made modifications to the text.

\section{Funding}

This study is supported by The Scientific and Technological Research Council of Turkey (TUBITAK), 1001Primary Subjects R\&D Funding Program, Project No: 117S145.

\section{Availability of data and materials}

All data is available through the senior author

\section{Ethics approval and consent to participate}

Written informed consent for the collection, storage and use of cells for research purposes were obtained and the study is approved by the Institutional Review Board of Hacettepe University (Study approval number: G0 16/562-08).

\section{Consent for publication}

Not applicable.

\section{Competing interest}

The authors declare no competing interests.

\section{Author details}


${ }^{1}$ Department of Stem Cell Sciences, Institute of Health Sciences, Hacettepe University, Ankara, Turkey.

${ }^{2}$ Center for Stem Cell Research and Development PEDI-STEM, Hacettepe University, Ankara, TURKEY

${ }^{3}$ Department of Pediatrics, Division of Pediatric Hematology and Bone Marrow Transplantation Unit, Faculty of Medicine, Hacettepe University, Ankara, TURKEY.

\section{References}

1. Sobacchi, C., et al., Osteopetrosis: genetics, treatment and new insights into osteoclast function. Nature Reviews Endocrinology, 2013. 9(9): p. 522.

2. Frattini, A., et al., Defects in TCIRG1 subunit of the vacuolar proton pump are responsible for a subset of human autosomal recessive osteopetrosis. Nature genetics, 2000. 25(3): p. 343-346.

3. Palagano, E., et al., Genetics of osteopetrosis. Current osteoporosis reports, 2018. 16(1): p. 13-25.

4. Zirngibl, R.A., et al., Novel c. G630A TCIRG1 mutation causes aberrant splicing resulting in an unusually mild form of autosomal recessive osteopetrosis. Journal of cellular biochemistry, 2019.

5. Orchard, P.J., et al., Hematopoietic stem cell transplantation for infantile osteopetrosis. Blood, 2015. 126(2): p. 270-276.

6. Del Fattore, A., M. Capannolo, and N. Rucci, Bone and bone marrow: the same organ. Archives of biochemistry and biophysics, 2010. 503(1): p. 28-34.

7. Teti, A., Osteoclasts and hematopoiesis. BoneKEy reports, 2012. 1.

8. Blin-Wakkach, C., M. Rouleau, and A. Wakkach, Roles of osteoclasts in the control of medullary hematopoietic niches. Archives of biochemistry and biophysics, 2014. 561: p. 29-37.

9. Winkler, I.G., et al., Bone marrow macrophages maintain hematopoietic stem cell (HSC) niches and their depletion mobilizes HSCs. Blood, 2010. 116(23): p. 4815-4828.

10. Mansour, A., et al., Osteoclasts promote the formation of hematopoietic stem cell niches in the bone marrow. Journal of Experimental Medicine, 2012. 209(3): p. 537-549.

11. Jacome-Galarza, C.E., et al., Developmental origin, functional maintenance and genetic rescue of osteoclasts. Nature, 2019. 568(7753): p. 541-545.

12. Adams, G.B., et al., Stem cell engraftment at the endosteal niche is specified by the calcium-sensing receptor. Nature, 2006. 439(7076): p. 599-603.

13. Kollet, $0 .$, et al., Osteoclasts degrade endosteal components and promote mobilization of hematopoietic progenitor cells. Nature medicine, 2006. 12(6): p. 657-664.

14. Lymperi, S., et al., Inhibition of osteoclast function reduces hematopoietic stem cell numbers in vivo. Blood, 2011. 117(5): p. 1540-1549.

15. Peled, A., et al., Dependence of human stem cell engraftment and repopulation of NOD/SCID mice on CXCR4. Science, 1999. 283(5403): p. 845-848. 
16. Kollet, O., A. Dar, and T. Lapidot, The multiple roles of osteoclasts in host defense: bone remodeling and hematopoietic stem cell mobilization. Annu. Rev. Immunol., 2007. 25: p. 51-69.

17. Mansour, A., A. Wakkach, and C. Blin-Wakkach, Role of osteoclasts in the hematopoietic stem cell niche formation. Cell Cycle, 2012. 11(11): p. 2045-2046.

18. Lymperi, S., et al., Inhibition of osteoclast function reduces hematopoietic stem cell numbers in vivo. Blood, The Journal of the American Society of Hematology, 2011. 117(5): p. 1540-1549.

19. Hartung, O., et al., Clump passaging and expansion of human embryonic and induced pluripotent stem cells on mouse embryonic fibroblast feeder cells. Current protocols in stem cell biology, 2010. 14(1): p. 1C. 10.1-1C. 10.15.

20. Grigoriadis, A.E., et al., Directed differentiation of hematopoietic precursors and functional osteoclasts from human ES and iPS cells. Blood, The Journal of the American Society of Hematology, 2010. 115(14): p. 2769-2776.

21. Livak, K.J. and T.D. Schmittgen, Analysis of relative gene expression data using real-time quantitative PCR and the 2- $\triangle \triangle C T$ method. methods, 2001. 25(4): p. 402-408.

22. Blin-Wakkach, C., et al., Hematological defects in the oc/oc mouse, a model of infantile malignant osteopetrosis. Leukemia, 2004. 18(9): p. 1505-1511.

23. Blin-Wakkach, C., et al., Interleukin-7 partially rescues B-lymphopoiesis in osteopetrotic oc/oc mice through the engagement of $B 220+C D 11 b+$ progenitors. Experimental hematology, 2006. 34(7): $p$. 851-859.

24. Del Fattore, A., A. Cappariello, and A. Teti, Genetics, pathogenesis and complications of osteopetrosis. Bone, 2008. 42(1): p. 19-29.

25. Mansour, A., et al., Osteoclast activity modulates B-cell development in the bone marrow. Cell research, 2011. 21(7): p. 1102-1115.

26. Matsubara, T., et al., Regulation of osteoclast differentiation and actin ring formation by the cytolinker protein plectin. Biochemical and biophysical research communications, 2017. 489(4): p. 472-476.

27. Kim, J.H. and N. Kim, Regulation of NFATc1 in osteoclast differentiation. Journal of bone metabolism, 2014. 21(4): p. 233.

28. Ferlin, A., et al., Relaxin stimulates osteoclast differentiation and activation. Bone, 2010. 46(2): p. 504-513.

29. Chen, W., et al., TCIRG1 transgenic rescue of osteoclast function using induced pluripotent stem cells derived from patients with infantile malignant autosomal recessive osteopetrosis. JBJS, 2019. 101(21): p. 1939-1947.

30. Cheng, H., Z. Zheng, and T. Cheng, New paradigms on hematopoietic stem cell differentiation. Protein \& cell, 2020. 11(1): p. 34-44.

31. Lu, R., et al., Clonal-level lineage commitment pathways of hematopoietic stem cells in vivo. Proceedings of the National Academy of Sciences, 2019. 116(4): p. 1447-1456. 
32. Singh, P., K.S. Mohammad, and L.M. Pelus, CXCR4 expression in the bone marrow microenvironment is required for hematopoietic stem and progenitor cell maintenance and early hematopoietic regeneration after myeloablation. Stem Cells, 2020. 38(7): p. 849-859.

33. Cao, $\mathrm{H}$. , et al., Osteopontin is an important regulative component of the fetal bone marrow hematopoietic stem cell niche. Cells, 2019. 8(9): p. 985.

34. Stegge, M., Interactions between hematopoietic stem cells and their niche. 2020.

35. Tamma, R. and D. Ribatti, Bone niches, hematopoietic stem cells, and vessel formation. International journal of molecular sciences, 2017. 18(1): p. 151.

36. Lee, D., D.W. Kim, and J.-Y. Cho, Role of growth factors in hematopoietic stem cell niche. Cell biology and toxicology, 2020. 36(2): p. 131-144.

37. Zhao, M., et al., $\mathrm{N}$-cadherin-expressing bone and marrow stromal progenitor cells maintain reserve hematopoietic stem cells. Cell reports, 2019. 26(3): p. 652-669. e6.

\section{Tables}

Table 1 not available with this version

\section{Figures}
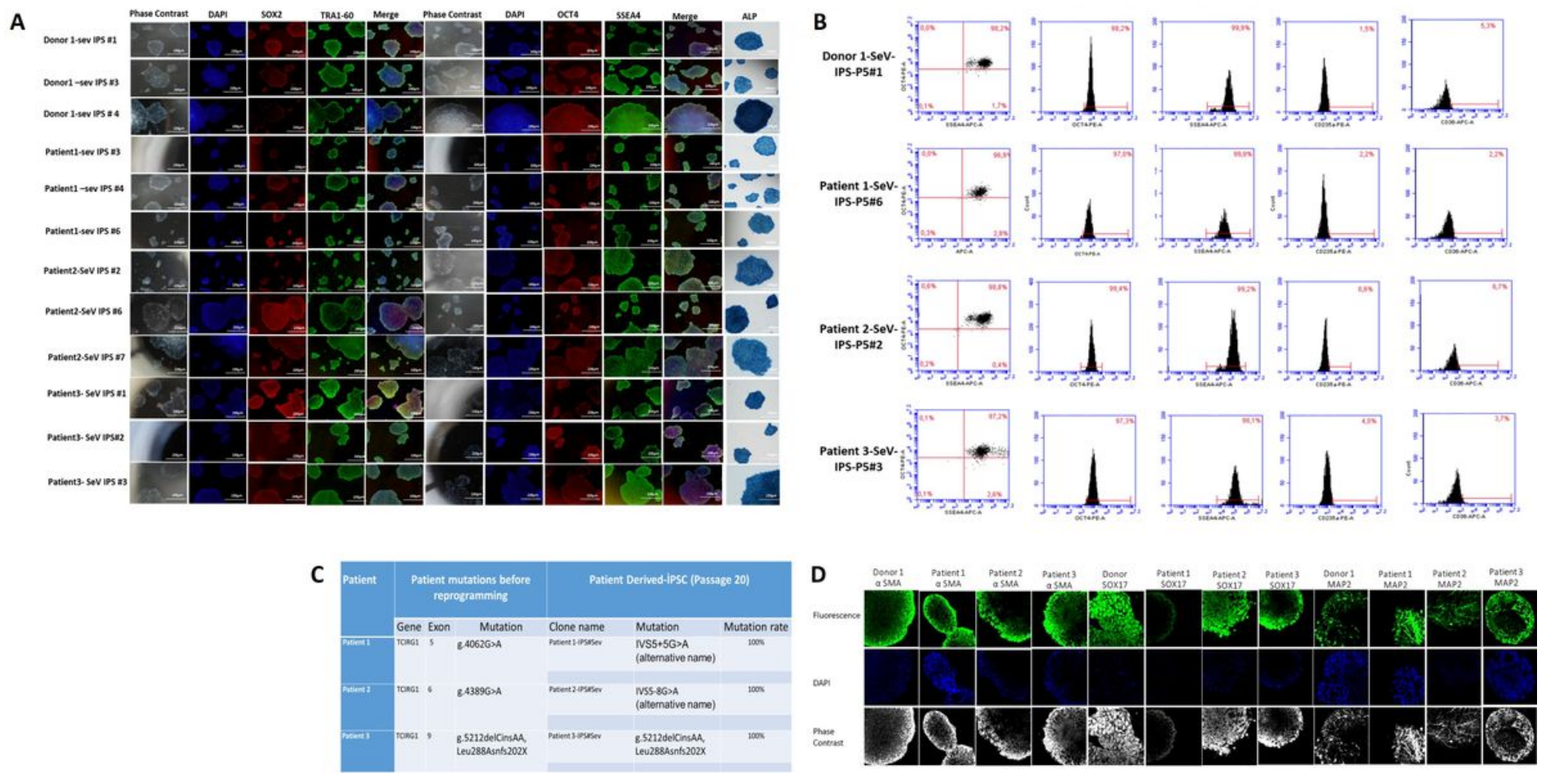

\section{Figure 1}

Characterization of established iPSC lines derived from patient and donor peripheral blood erythroid precursors. a) Immunofluorescence and ALP staining of three different clones of each of the selected 
iPSCs lines ( $n=12)$. b) Flow cytometry analysis with pluripotency markers, OCT4, SSEA4 and erythroid progenitor cell surface markers, CD36 and CD235a after reprogramming $(n=12)$. c) Verification of persistence of three different TCIRG1 mutations in osteopetrotic iPSCs ( $n=3)$. d) Whole-Mount immunostaining of spontaneously differentiated embryoid bodies with lineage-specific markers; MAP2 (ectoderm), a-SMA (mesoderm), SOX17 (endoderm) $(n=4)$.
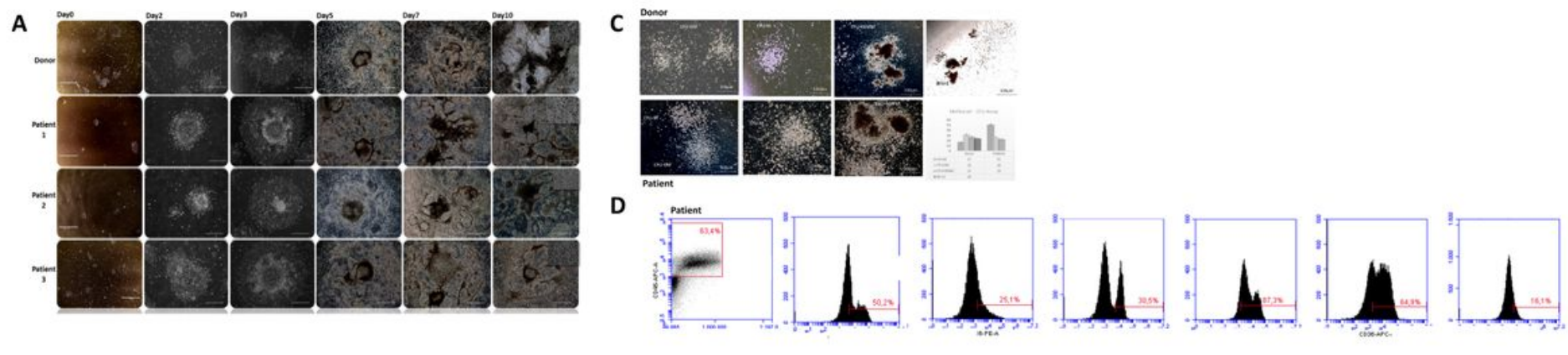

B
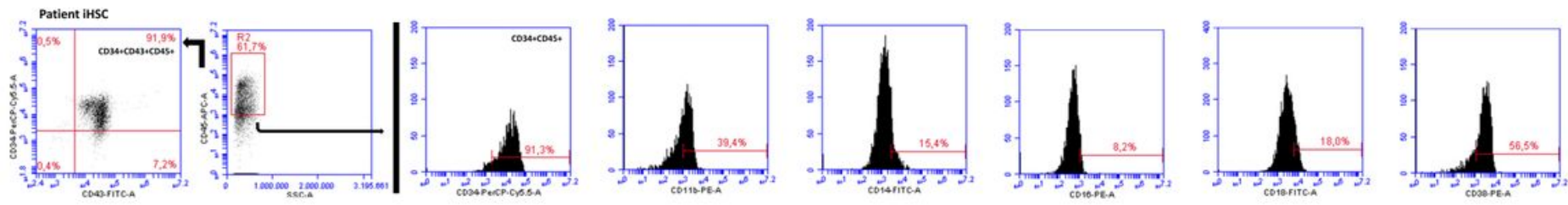

E
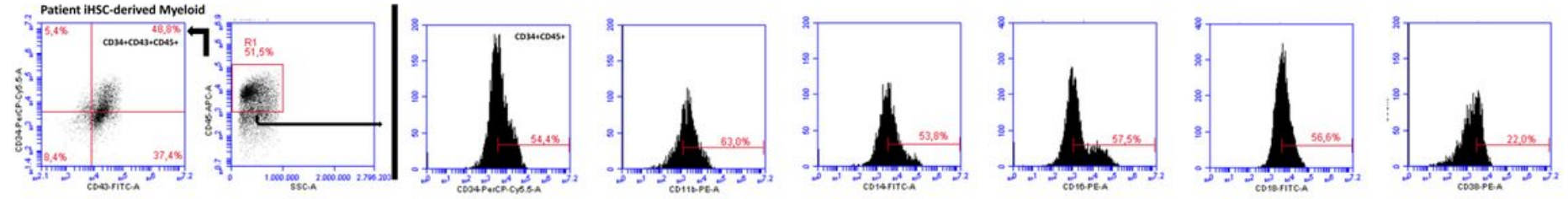

$\mathbf{F}$
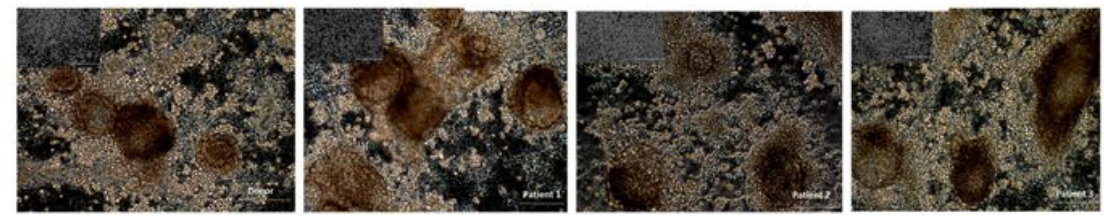

Figure 2

Assessment of hematopoietic differentiation potential of iPSC lines. a) Differentiated blood-forming iHSC colonies with the formation of radial sac-like structures. b) Flow cytometry analyses of osteopetrotic iPSCs that are differentiated to hematopoietic progenitor cells as indicated by an increase in CD34+CD 45+ cell fraction by day 10 following hematopoietic induction coupled with low expression of myelomonocytic markers (CD14, CD16, CD18, 11b, CD38, and CD43). Hematopoietic differentiation potential of patient -and donor-derived iPSCs was evaluated with unpaired two-tailed T test. C) Confirmation of the clonogenic potential of iHSCs by Colony Forming Unit assay Donor iPSCs derived iHSCs colonies (upper row), patient iPSCs derived iHSCs colonies (lower row), the mean number of different types of hematopoietic colonies were given at the right corner. d) Confirmation of hematopoietic lineage commitment of cells forming colonies by flow cytometry after methocult assay. e) Flow cytometry analysis of myeloid differentiated osteopetrotic iHSCs at Day 14 as indicated by an increase in myelomonocytic cell surface markers following myeloid induction (Unpaired two-tailed T test for comparison of patient and donor iPSCs derived myeloid cells, paired two-tailed T test for comparison of 
patient-iHSCs and patient iHSCs derived myeloid cells. P<0.05). f) Morphological evaluation of myeloid differentiated cells (left-top 10x magnification, main figure 4x magnification).
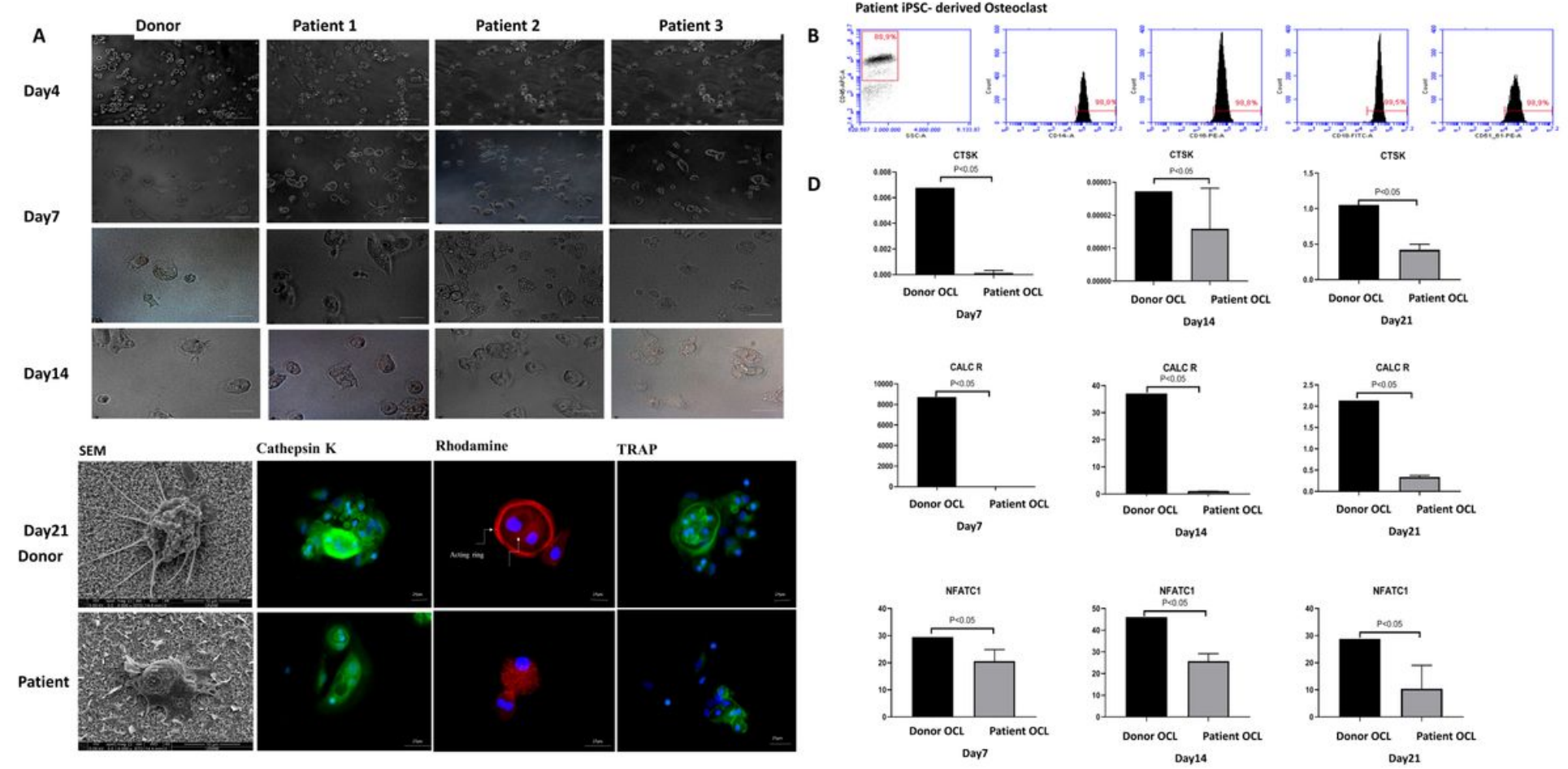

Figure 3

Differentiation of osteoclasts from iPSC-derived myelomonocytic precursors a) Morphological evaluation of iPSCs derived osteoclasts. Morphological changes were observed in the cells throughout the differentiation process (50 $\mu \mathrm{m}$ magnification). b) A representative of flow cytometry analysis of osteoclasts derived from iPSCs indicates an increase in osteoclast progenitor cell markers (CD14, CD16, CD18, CD45, CD51/6). c) SEM imaging of iPSCs derived-osteoclasts ( $10 \mu \mathrm{m}$ magnification), and immunofluorescence staining (Cathepsin K, Rhodamine, TRAP) of functional osteoclasts $(25 \mu \mathrm{m}$ magnification) for comparison of osteopetrotic - and donor-derived cells (upper row-donor, lower rowpatient). d) qRT-PCR analysis for osteoclast-specific CTSK, CALCR, and NFATC1 gene expression (3 Patients, 1 donor). One sample Wilcoxon test was performed one by one for Day7, Day14, and Day21 ( $P<$ 0.05), 
A
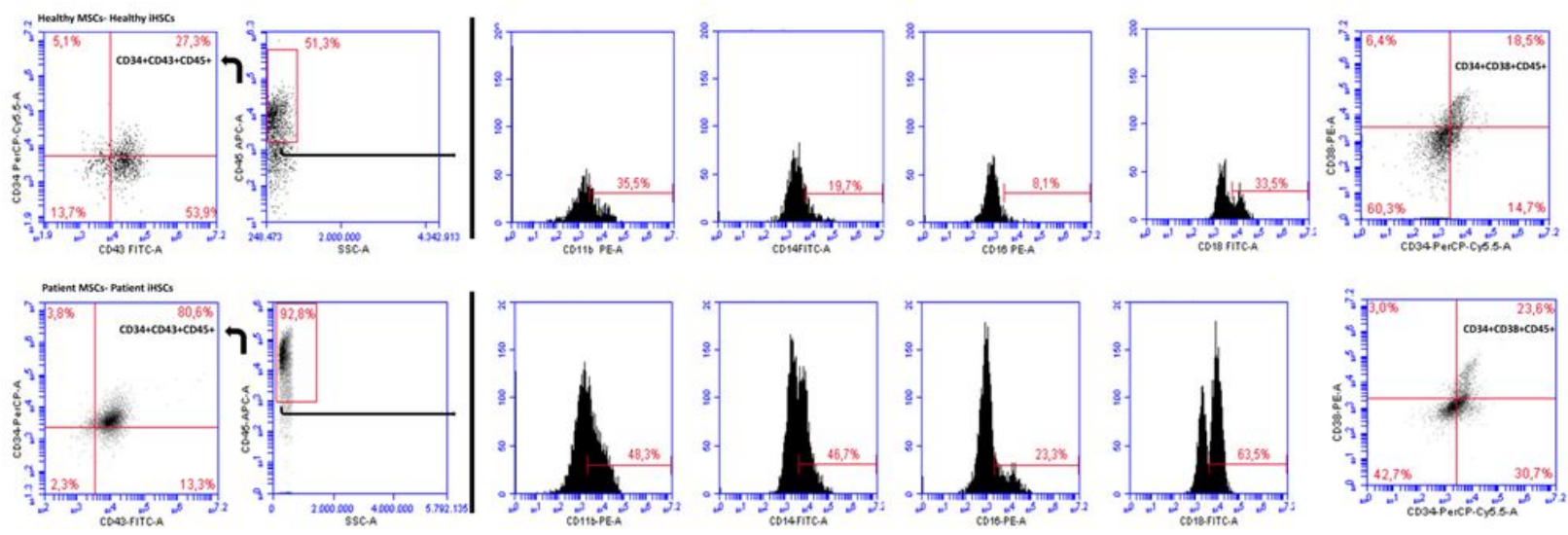

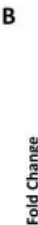

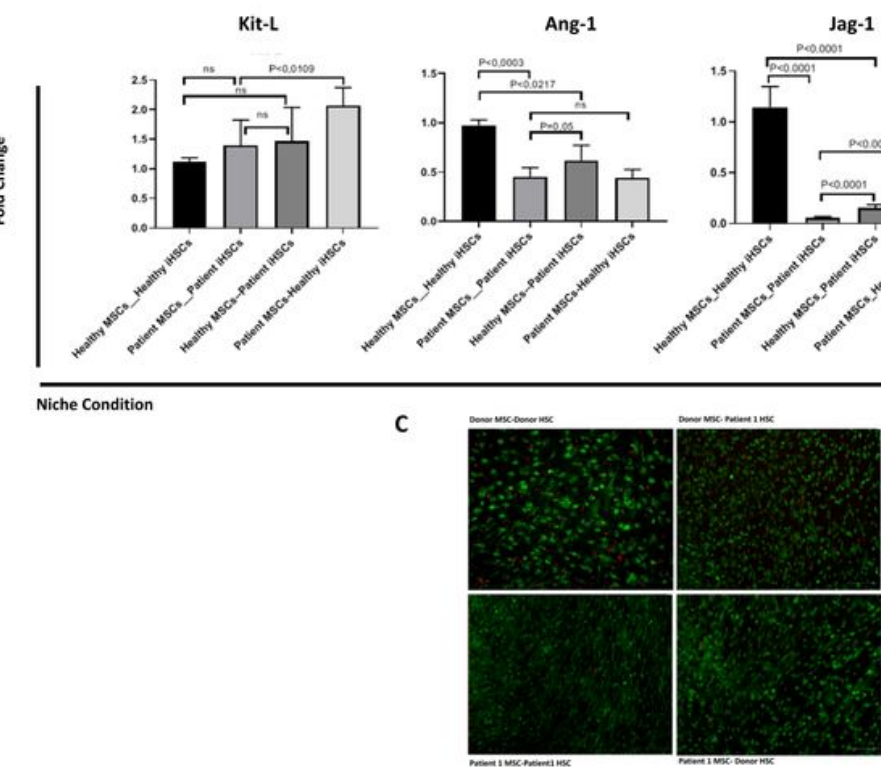

Sdf-1
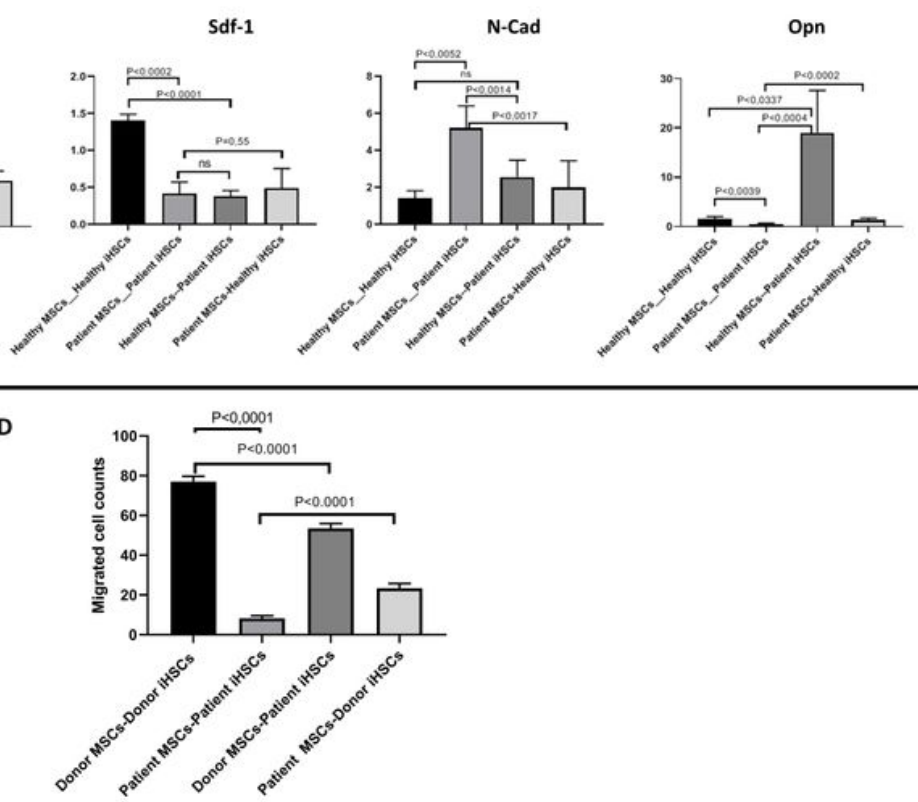

Figure 4

Co-culture of BM-MSCs and iHSCs; evaluation of immunophenotype, migration potential of iHSCs and gene expression profile of BM-MSCs a) Representative figure shows alteration of hematopoietic and myeloid-lineage specific surface markers after co-culture (upper row- healthy MSCs-healthy iHSCs, lower row -patient MSCs-patient iHSCs) (Unpaired two-tailed T test; $\mathrm{P}<0.05)$. b) Analysis of expression of the genes involved in the regulation of HSCs homing and maintenance in the hematopoietic niche; Sdf-1, Jagged-1, N-Cad, Ang-1, Kit-L, Opn in donor and osteopetrotic MSCs after co-culture with qRT-PCR (Unpaired two-tailed $T$ test was used for comparison of each condition, $P<0.05$ ).c) Representative figure shows migration potential of osteopetrotic and healthy iHSCs. d) Bar graph shows mean number of migrated HSCs (Unpaired two-tailed T test was used for comparison of each condition, $\mathrm{P}<0.05$ ).

\section{Supplementary Files}

This is a list of supplementary files associated with this preprint. Click to download.

- SupplementaryFigure1.jpg 
- SupplementaryFigure2.jpg

- SupplementaryFigure3.jpg

- SupplementaryFigure4.jpg

- SupplementaryTable1.Resourcetable.docx

- Graphicalabstract.jpg 